\title{
High Prevalence of Late-Appearing T-Wave in Patients With Long QT Syndrome Type 8
}

\author{
Megumi Fukuyama, MD, PhD; Seiko Ohno, MD, PhD; Junichi Ozawa, MD, PhD; \\ Koichi Kato, MD, PhD; Takeru Makiyama, MD, PhD; \\ Yoshihisa Nakagawa, MD, PhD; Minoru Horie, MD, PhD
}

\begin{abstract}
Background: Long QT syndrome type 8 (LQT8) is a rare genotype of long QT syndrome. Late-appearing T-waves (LaT) are often documented in patients with LQT8, as in long QT syndrome type 3 (LQT3); however, the frequency of LaT and its relevance to the clinical severity of LQT8 remains unclear. This study investigated T-wave morphology (TWM) in LQT3 and LQT8 patients and compared the phenotypes of different TWMs.
\end{abstract}

\begin{abstract}
Methods and Results: TWMs were classified into 3 types: early onset T-waves (EOT), LaT, and bifid T-waves (biT). Electrocardiogram (ECG) measurements, symptoms, and topology were compared among TWM types. The study cohort comprised 25 patients with LQT8 (14 mutations) and 25 patients with LQT3 (14 mutations). LaT was detected in 17 (68\%) and 13 (52\%) LQT8 and LQT3 patients, respectively. There were no significant differences in ECG measurements or the severity of symptoms between patients with LaT and those with other TWMs in either the LQT8 or LQT3 group. However, only patients with LaT experienced cardiopulmonary arrest. Compared with the LQT3 group, in the LQT8 group there was a tendency for mutations in patients with LaT to be located in domain-linking regions.
\end{abstract}

Conclusions: In this study, two-thirds of patients with LQT8 exhibited LaT on ECG, and nearly one-third of those experienced cardiopulmonary arrest. Further investigations are warranted to differentiate between LQT3 and LQT8 in patients exhibiting LaT to optimize therapy.

Key Words: Genetics; Late-appearing T-wave; Long QT syndrome; LQT8; T-wave morphology

$\mathbf{O}$ $\mathrm{n}$ an electrocardiogram (ECG), congenital long QT syndrome (LQTS) presents not only as QT prolongation, but also as abnormal $\mathrm{T}$-wave morphology (TWM). ${ }^{1}$ LQTS is genetically diverse, with causative mutations in >10 genes; however, most patients harbor mutations in 3 major genes: potassium voltage-gated channel subfamily Q member 1 (KCNQ1), potassium voltage-gated channel subfamily $\mathrm{H}$ member $2(\mathrm{KCNH})$, and sodium voltage-gated channel $\alpha$ subunit $5\left(\right.$ SCN5A). ${ }^{2-4}$ In the very early stage of LQTS genetic screening, T-wave patterns were found to differ based on the genotype; reportedly, late-appearing T-wave (LaT) is a hallmark of carriers of $S C N 5 A$ mutations (i.e., those with long QT type 3 [LQT3]) ${ }^{\mathbf{5}, \mathbf{6}}$

Long QT syndrome type 8 (LQT8), or Timothy syndrome (TS), is caused by mutations in calcium voltage-gated channel subunit $\alpha 1 \mathrm{C}(C A C N A 1 C)$, which encodes the Cav1.2 protein, the $\alpha$-subunit of the voltage-gated $\mathrm{Ca}$ channel protein. TS, a systemic disease with excessive QT prolongation, ${ }^{7}$ is a rare subtype of LQTS that has several phenotypes and is associated with early mortality. The diverse extracardiac phenotypes of TS include multiple organ systems (i.e., congenital heart disease, syndactyly, immune deficiency, dysfunction of the central nervous system, and metabolic abnormalities), justifying its separate classification as a disease entity. Regarding TS-associated mutations, only $2 C A C N A 1 C$ mutations (p.G402S and p.G406R) have been reported since its first discovery in 2004. ${ }^{78}$ In 2014, we reported 7 LQT8 probands with CACNA1C mutations among 278 LQTS probands and established that the frequency of LQT8 was higher than thought and that $C A C N A I C$ mutation-related clinical phenotypes other than those related to TS are mild and lack extracardiac phenotypes. ${ }^{9}$ Moreover, recent advances in genetic analyses revealed that LQT8 is not too rare as classical TS. ${ }^{10-15}$

Received December 5, 2019; revised manuscript received January 22, 2020; accepted February 4, 2020; J-STAGE Advance Publication released online March 12, 2020 Time for primary review: 32 days

Department of Cardiovascular Medicine (M.F., K.K., Y.N., M.H.), Center for Epidemiologic Research in Asia (M.H.), Shiga University of Medical Science, Otsu; Department of Bioscience and Genetics, National Cerebral and Cardiovascular Center, Suita (S.O.); Department of Pediatrics, Niigata University Graduate School of Medical and Dental Sciences, Niigata (J.O.); and Department of Cardiovascular Medicine, Kyoto University Graduate School of Medicine, Kyoto (T.M.), Japan

Mailing address: Seiko Ohno, MD, PhD, Department of Bioscience and Genetics, National Cerebral and Cardiovascular Center, 6-1 Kishibeshinmachi, Suita 564-8565, Japan. E-mail: sohno@ncvc.go.jp

ISSN-1346-9843 All rights are reserved to the Japanese Circulation Society. For permissions, please e-mail: cj@j-circ.or.jp 


\section{(A) Early-onset T-wave (EoT)}

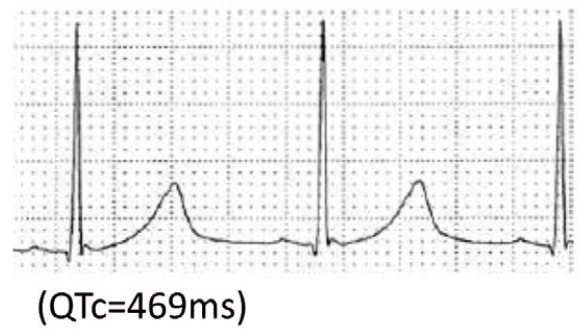

(B) Late-appearance T-wave (LaT)

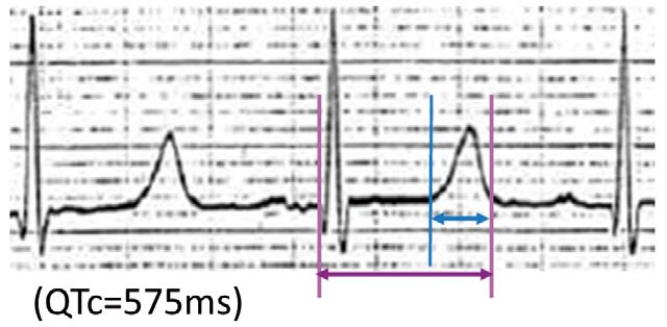

\section{(C) Bifid T-wave (biT)}

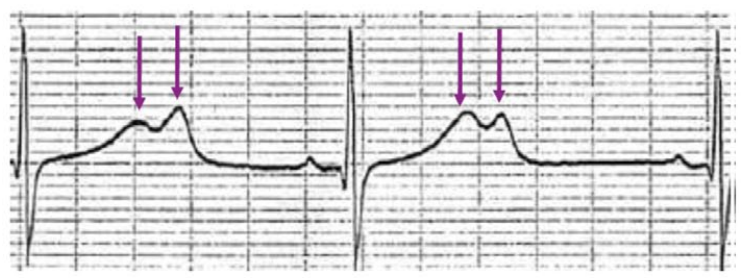

(QTc=565ms)

Figure 1. Definition of T-wave morphology. (A) Early onset T-waves (EoT). The starting point of the T-wave is just after the QRS complex with smooth transition from the ST segment. (B) Late-appearing T-wave (LaT). The starting point of the T-wave is after more than half the QT duration. (C) Bifid T-waves (biT) are those waves that are not LaT and have 2 peaks or notches (arrows) in at least 1 precordial lead. QTc, corrected QT interval.

Conversely, LQT3 is caused by mutations of $S C N 5 A$, which encodes the Nav1.5 protein, the $\alpha$-subunit of the voltage-gated $\mathrm{Na}^{+}$channel. Structurally, the Cav1.2 and Nav1.5 proteins are similar. ${ }^{16-18}$ Both Cav1.2 and Nav1.5 channels carry inward depolarizing currents, and gain-offunction mutations in their encoding genes cause late inward currents during the plateau phase of the action potential, and thus LQTS.

Because 4 years have passed since our first report on LQT8, ${ }^{9}$ and our LQTS database has increased from 568 to 892 probands, we now have approximately 3-fold more $C A C N A 1 C$ mutation carriers (LQT8 patients). A comprehensive ECG analysis revealed a higher frequency of LaT in these LQT8 patients. ${ }^{19}$ However, the precise frequency of LaT, its relevance to the clinical severity, and its relationship with the location of mutations in LQT8 remain unclear. Hence, the aims of this study were to determine TWM patterns in patients with LQT8, to correlate these patterns with the different phenotypes and to compare the findings with those in LQT3 patients.

\section{Methods}

\section{Patient Cohort}

In all, 892 Japanese LQTS probands who were registered at the Shiga University of Medical Science (Otsu, Japan) or the Kyoto University Graduate School of Medicine (Kyoto, Japan) from 1996 to 2017 were enrolled in this study and underwent genetic analysis. Written informed consent was obtained from all patients as per the guidelines or each institutional review board.

The criteria for patient selection were based on those in our previous study. ${ }^{9}$ Briefly, LQTS was suspected upon the appearance of 1 of the following: QT prolongation in a 12-lead ECG and/or personal history of syncope; fatal arrhythmic attacks; cardiopulmonary arrest (CPA); and severe bradycardia for age. ${ }^{20} \mathrm{QT}$ intervals were measured manually in Leads II or V5. ${ }^{4}$ Corrected QT intervals (QTc), corrected for heart rate, were evaluated using Bazett's formula. Briefly, the ECG criteria for QTc intervals are $\geq 450$ and $\geq 460 \mathrm{~ms}$ for males and females, respectively. ${ }^{\mathbf{2 1}}$

TWM was classified into 3 types: early onset of T-waves with smooth transition from the ST segment (EoT; Figure 1A), LaT (Figure 1B), and bifid T-waves (biT; Figure 1C). If the starting point of the $\mathrm{T}$-wave was just after the QRS morphology with a prolonged QT interval, the T-wave was defined as EoT; LaT was defined as a T-wave with a start pointing after more than half the QT duration, and biT was defined as a T-wave that did not show LaT but had 2 peaks or notches in at least 1 of the precordial leads. For TWM analysis, probands who harbored other causative mutations of LQTS or right/left bundle branch block, or inverted T-wave were excluded. There were no day-to-day changes in TWM observed on multiple ECGs from the same patients.

In each TWM group, ECG measurements, symptoms, and the locations of mutations were examined, with the findings compared between groups as well as with LQT3 patients.

This study was approved by the Institutional Review Board of Shiga University of Medical Science (Reference no. 23-128-3).

\section{Genetic Screening}

Genomic DNA was extracted from peripheral blood leukocytes. In 2011-2014, gene screening was performed using the high-resolution melting method (HRM) 22 and/or subsequent direct sequencing. ${ }^{23}$ Samples were screened for the causative genes of LQT1-8 (KCNQ1, KCNH2, SCN5A, ankyrin $2[A N K 2]$, potassium voltage-gated channel subfamily E regulatory subunit 1/2 [KCNE1/2], potassium inwardly rectifying channel subfamily $\mathrm{J}$ member 2 [KCNJ2], and $C A C N A 1 C$ ). Since 2015, 56 genes associated with inherited primary arrhythmia syndromes, including LQTSrelated genes, have been screened for using the HaloPlex HS custom panel (Agilent Technologies, Palo Alto, CA, USA) and a bench top-type next-generation sequencer (MiSeq; Illumina, San Diego, CA, USA). Data were analyzed using SureCall software (Agilent Technologies). Any variants detected were confirmed using the Sanger method. ${ }^{23}$ In the case of $C A C N A 1 C$, the number of nucleotides was based on NM_000719.

Variants were classified based on their pathogenicity according to the 2015 American College of Medical Genetics and Genomics (ACMG) standards and guidelines ${ }^{24}$ as pathogenic, likely pathogenic, or uncertain significance. 


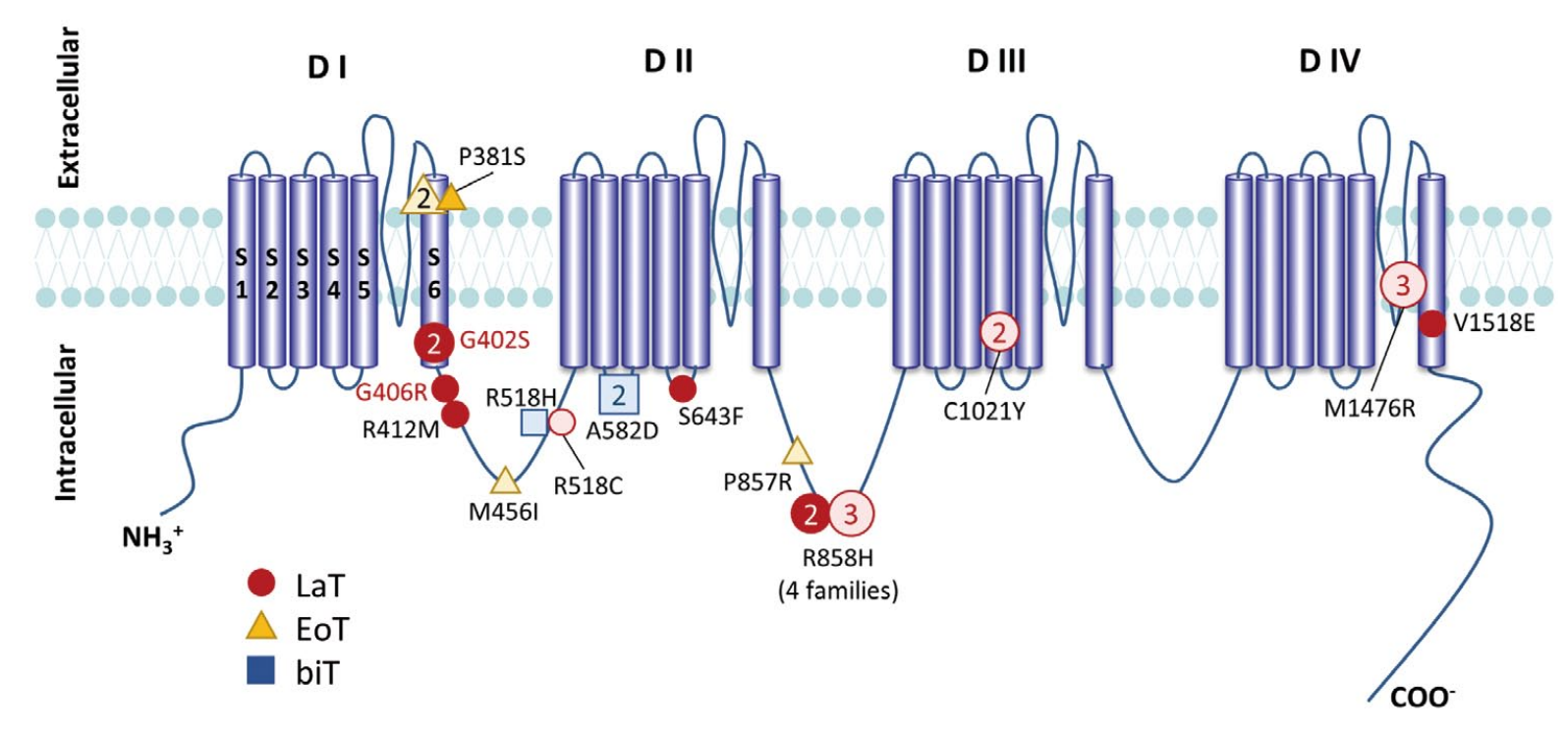

Figure 2. Locations of mutations in Cav1.2. LaT, late-appearing T-wave; EoT, early onset T-wave; biT, bifid T-wave. Numbers inside symbols indicate the number of carriers; solid symbols indicate symptomatic patients and open symbols indicate asymptomatic patients.

\section{Topology}

Cav1.2 and Nav1.5 have a similar structure, ${ }^{\mathbf{1 6}, 25}$ with their $\alpha$-subunits having 4 repeat domains (DI-DIV). Each domain comprises 6 membrane-spanning segments (S1-S6). The ions are conducted through a "pore" formed by the combined S5 and S6 segments. The region connecting domains is defined as the D-linker (Figure 2).

\section{Statistical Analysis}

Continuous clinical data are presented as the mean \pm SD. The significance of between-group differences was evaluated using independent Student's t-tests. Two-tailed $\mathrm{P}<0.05$ was considered significant.

\section{Results}

\section{Clinical and Genetic Characteristics of LQT8 and LQT3 Mutation Carriers}

Among 892 LQTS probands, 17 LQT8 and 20 LQT3 probands were genotyped. Table 1 summarizes the clinical and genetic characteristics of the LQT8 cohort, which comprised 25 patients (14 mutations; mean age at diagnosis $16.0 \pm 14.8$ years), including 8 family members. The mean heart rate (HR) of this group was $70 \pm 18$ beats $/ \mathrm{min}$; the mean QTc interval was $521 \pm 86 \mathrm{~ms}$, the mean QT peak (QTp) was $390 \pm 80 \mathrm{~ms}$, and the T-wave peak to end (Tpe) was $101 \pm 25 \mathrm{~ms}$. Notably, 3 patients had experienced CPA, 2 had torsades de pointes (TdP), and 4 had syncope. Furthermore, $9(64 \%)$ mutations were located in the D-linker region, 4 were in the transmembrane region, and 1 was in the pore region. Among the $14 C A C N A 1 C$ variants, there were 10 "pathogenic" and 4 "likely pathogenic" variants (Table 1).

Table 2 summarizes the clinical features of the 20 LQT3 probands and 5 genotype-positive family members (14 mutations; mean age at diagnosis $13.0 \pm 12.3$ years). The mean HR in this group was $78 \pm 25$ beats $/ \mathrm{min}$; the mean
QTc interval was $530 \pm 72 \mathrm{~ms}$, the mean QTp was $379 \pm 89 \mathrm{~ms}$, and the mean Tpe was $97 \pm 25 \mathrm{~ms}$. Of these patients, 2 had experienced CPA, 3 had TdP, 4 had syncope, and 1 had a transient functional 2:1 atrioventricular block. There were no significant differences in ECG parameters between the LQT8 and LQT3 groups (Table 3). Of the 14 SCN5A variants in the LQT3 group, 6 were "pathogenic", 6 were "likely pathogenic", and 2 were of "uncertain significance" (Table 2).

\section{Comparison of Clinical Features Among TWM Groups}

Among the patients with LQT8, LaT was detected in 17 $(68 \%)$, EoT was detected in $5(20 \%)$, and biT was detected in $3(12 \%)$. Among those with LQT3, the number of EoT and LaT was similar $(\mathrm{n}=11$ [44\%] and 13 [52\%], respectively), with the remaining patients having biT (Figure 3A).

Patients with LQT8 were divided into 3 groups based on TWM (EoT, LaT, and biT). The mean age at diagnosis in the EoT, LaT, and biT groups was 17.2 $\pm 13.1,14.4 \pm 15.3$, and 23.0 \pm 17.3 years, respectively; the mean HR in these 3 groups was $65 \pm 9,75 \pm 18$, and $49 \pm 6$ beats/min, respectively, and the mean QTc interval was 458 $\pm 32,533 \pm 90$, and $556 \pm 93 \mathrm{~ms}$, respectively (Table 3). The mean age, HR, and QTc intervals for patients with LQT3 were similar to those with LQT8, except for patients with biT. For patients with LQT3, the mean age at diagnosis was 15.6 $\pm 12.2,11.4 \pm 12.8$, and 6 years in the EoT, LaT, and biT groups. The mean $\mathrm{HR}$ in the EoT, LaT, and biT groups was $78 \pm 21,75 \pm 27$, and 115 beats/min, respectively, and the mean QTc interval was $502 \pm 53,557 \pm 80$, and $499 \mathrm{~ms}$, respectively (Table 3). In both the LQT3 and LQT8 groups, there were no significant differences in the QTc interval among the 3 TWM groups (Figure 3B). Similarly, there were no significant differences in QTp and Tpe between the LQT8 and LQT3 groups according to TWM (Figure 3C,D; Table 3).

Of the 9 symptomatic LQT8 patients, 8 (89\%) exhibited the LaT pattern (3 CPA, $2 \mathrm{TdP}$, and 3 syncope [ventricular 


\begin{tabular}{|c|c|c|c|c|c|c|c|c|c|c|c|c|c|c|}
\hline $\begin{array}{l}\text { Patient } \\
\text { no. }\end{array}$ & Status & $\begin{array}{c}\text { Age } \\
\text { (years) }\end{array}$ & Sex & $\begin{array}{c}\text { HR } \\
\text { (beats/ } \\
\text { min) }\end{array}$ & $\begin{array}{l}\text { QTp } \\
\text { (ms) }\end{array}$ & $\begin{array}{l}\text { Tpe } \\
\text { (ms) }\end{array}$ & $\begin{array}{l}\text { QTc } \\
\text { (ms) }\end{array}$ & TWM & Symptom & $\begin{array}{l}\text { Nucleotide } \\
\text { change }\end{array}$ & $\begin{array}{l}\text { Amino } \\
\text { acid } \\
\text { change }\end{array}$ & Location & $\begin{array}{l}\text { Pathoge- } \\
\text { nicity }\end{array}$ & $\begin{array}{c}\text { Func- } \\
\text { tional } \\
\text { proper- } \\
\text { ties }\end{array}$ \\
\hline 1 & Pro & 10 & $\mathrm{~F}$ & 65 & 380 & 60 & 469 & EоT & $\begin{array}{l}\text { Asymp- } \\
\text { tomatic }\end{array}$ & c. $1141 \mathrm{c}>\mathrm{t}$ & P381S & DI S6 & LP & NS \\
\hline 2 & Fam & 39 & $M$ & 61 & 360 & 80 & 444 & EoT & $\begin{array}{l}\text { Asymp- } \\
\text { tomatic }\end{array}$ & c. $1141 \mathrm{c}>\mathrm{t}$ & P381S & DI S6 & LP & NS \\
\hline 3 & Fam & 19 & $\mathrm{~F}$ & 53 & 370 & 70 & 414 & Еот & Syncope & c. $1141 \mathrm{c}>\mathrm{t}$ & P381S & DI S6 & LP & NS \\
\hline 4 & Pro & 10 & $\mathrm{~F}$ & 92 & 400 & 100 & 575 & LaT & $\begin{array}{l}\text { TdP/VF } \\
\text { storm }\end{array}$ & c. $1204 \mathrm{~g}>\mathrm{a}$ & G402S & DI S6 & $\mathrm{P}$ & GOF \\
\hline 5 & Pro & 0 & M & 69 & 560 & 100 & 708 & LaT & $\begin{array}{c}\text { TdP, } 2: 1 \\
\text { AVB }\end{array}$ & c. $1204 \mathrm{~g}>\mathrm{a}$ & G402S & DI S6 & $\mathrm{P}$ & GOF \\
\hline 6 & Pro & 0 & $\mathrm{~F}$ & 79 & 440 & 160 & 688 & LaT & Syncope & c. $1216 \mathrm{~g}>\mathrm{a}$ & G406R & $\begin{array}{l}\text { DI-DII } \\
\text { linker }\end{array}$ & $P$ & GOF \\
\hline 7 & Pro & 0 & $F$ & 88 & 430 & 130 & 679 & LaT & $\begin{array}{l}\text { CPA } \\
(\mathrm{TdP})\end{array}$ & c. $1235 \mathrm{~g}>\mathrm{t}$ & $\mathrm{R} 412 \mathrm{M}$ & $\begin{array}{l}\text { DI-DII } \\
\text { linker }\end{array}$ & $P$ & GOF \\
\hline 8 & Pro & 12 & $\mathrm{~F}$ & 73 & 340 & 100 & 465 & Еот & $\begin{array}{l}\text { Asymp- } \\
\text { tomatic }\end{array}$ & c. $1368 \mathrm{~g}>\mathrm{a}$ & M456I & $\begin{array}{l}\text { DI-DII } \\
\text { linker }\end{array}$ & LP & NS \\
\hline 9 & Pro & 7 & $M$ & 72 & 340 & 102 & 484 & LaT & $\begin{array}{l}\text { Asymp- } \\
\text { tomatic }\end{array}$ & c. $1552 c>t$ & R518C & $\begin{array}{l}\text { DI-DII } \\
\text { linker }\end{array}$ & $\mathrm{P}$ & N/A \\
\hline 10 & Pro & 14 & $M$ & 45 & 460 & 60 & 458 & biT & $\begin{array}{l}\text { Asymp- } \\
\text { tomatic }\end{array}$ & c. $1553 \mathrm{~g}>\mathrm{a}$ & $\mathrm{R} 518 \mathrm{H}$ & $\begin{array}{l}\text { DI-DII } \\
\text { linker }\end{array}$ & $P$ & N/A \\
\hline 11 & Pro & 12 & $F$ & 46 & 560 & 120 & 565 & biT & $\begin{array}{l}\text { Asymp- } \\
\text { tomatic }\end{array}$ & c. $1745 \mathrm{c}>\mathrm{a}$ & A582D & $\begin{array}{l}\text { DII S2- } \\
\text { S3 linker }\end{array}$ & $P$ & GOF \\
\hline 12 & Fam & 43 & $\mathrm{~F}$ & 56 & 560 & 100 & 644 & biT & $\begin{array}{l}\text { Asymp- } \\
\text { tomatic }\end{array}$ & c. $1745 \mathrm{c}>\mathrm{a}$ & A582D & $\begin{array}{l}\text { DII S2- } \\
\text { S3 linker }\end{array}$ & $P$ & GOF \\
\hline 13 & Pro & 13 & $M$ & 82 & 360 & 100 & 585 & LaT & CPA (VF) & c. $1928 c>t$ & S643F & $\begin{array}{l}\text { DII S4- } \\
\text { S5 linker }\end{array}$ & $P$ & GOF \\
\hline 14 & Pro & 6 & $\mathrm{~F}$ & 74 & 320 & 80 & 499 & ЕoT & $\begin{array}{l}\text { Asymp- } \\
\text { tomatic }\end{array}$ & c. $2570 \mathrm{c}>\mathrm{g}$ & P857R & $\begin{array}{l}\text { DII-DIII } \\
\text { linker }\end{array}$ & $P$ & GOF \\
\hline 15 & Pro & 15 & $M$ & 44 & 400 & 90 & 420 & LaT & Syncope & c. $2573 \mathrm{~g}>\mathrm{a}$ & $\mathrm{R} 858 \mathrm{H}$ & $\begin{array}{l}\text { DII-DIII } \\
\text { linker }\end{array}$ & $P$ & GOF \\
\hline 16 & Pro & 38 & M & 55 & 360 & 120 & 474 & LaT & $\begin{array}{l}\text { Asymp- } \\
\text { tomatic }\end{array}$ & c. $2573 \mathrm{~g}>\mathrm{a}$ & $\mathrm{R} 858 \mathrm{H}$ & $\begin{array}{l}\text { DII-DIII } \\
\text { linker }\end{array}$ & $P$ & GOF \\
\hline 17 & Pro & 7 & $M$ & 77 & 360 & 74 & 476 & LaT & $\begin{array}{c}\text { Syncope } \\
\text { (VT) }\end{array}$ & c. $2573 \mathrm{~g}>\mathrm{a}$ & $\mathrm{R} 858 \mathrm{H}$ & $\begin{array}{l}\text { DII-DIII } \\
\text { linker }\end{array}$ & $P$ & GOF \\
\hline 18 & Fam & 15 & M & 65 & 400 & 158 & 582 & LaT & $\begin{array}{l}\text { Asymp- } \\
\text { tomatic }\end{array}$ & c. $2573 \mathrm{~g}>\mathrm{a}$ & $\mathrm{R} 858 \mathrm{H}$ & $\begin{array}{l}\text { DII-DIII } \\
\text { linker }\end{array}$ & $P$ & GOF \\
\hline 19 & Fam & 40 & $\mathrm{~F}$ & 64 & 360 & 108 & 482 & LaT & $\begin{array}{l}\text { Asymp- } \\
\text { tomatic }\end{array}$ & c. $2573 \mathrm{~g}>\mathrm{a}$ & $\mathrm{R} 858 \mathrm{H}$ & $\begin{array}{l}\text { DII-DIII } \\
\text { linker }\end{array}$ & $P$ & GOF \\
\hline 20 & Pro & 37 & $\mathrm{~F}$ & 70 & 320 & 122 & 444 & LaT & $\begin{array}{l}\text { Asymp- } \\
\text { tomatic }\end{array}$ & c. $3062 \mathrm{~g}>\mathrm{a}$ & C1021Y & DIII S4 & LP & $\mathrm{N} / \mathrm{A}$ \\
\hline 21 & Fam & 11 & $F$ & 68 & 380 & 90 & 502 & LaT & $\begin{array}{l}\text { Asymp- } \\
\text { tomatic }\end{array}$ & c. $3062 \mathrm{~g}>\mathrm{a}$ & C1021Y & DIII S4 & LP & $\mathrm{N} / \mathrm{A}$ \\
\hline 22 & Pro & 47 & $\mathrm{~F}$ & 80 & 330 & 96 & 494 & LaT & $\begin{array}{l}\text { Asymp- } \\
\text { tomatic }\end{array}$ & c. $4427 t>g$ & M1476R & $\begin{array}{l}\text { DIV S5- } \\
\text { S6 pore }\end{array}$ & $P$ & N/A \\
\hline 23 & Fam & 33 & $F$ & 50 & 400 & 109 & 462 & LaT & $\begin{array}{l}\text { Asymp- } \\
\text { tomatic }\end{array}$ & c. $4427 \mathrm{t}>\mathrm{g}$ & M1476R & $\begin{array}{l}\text { DIV S5- } \\
\text { S6 pore }\end{array}$ & $P$ & N/A \\
\hline 24 & Fam & 5 & $F$ & 68 & 400 & 112 & 546 & LaT & $\begin{array}{l}\text { Asymp- } \\
\text { tomatic }\end{array}$ & c. $4427 \mathrm{t}>\mathrm{g}$ & M1476R & $\begin{array}{l}\text { DIV S5- } \\
\text { S6 pore }\end{array}$ & $P$ & $\mathrm{~N} / \mathrm{A}$ \\
\hline 25 & Pro & 0 & M & 128 & 240 & 95 & 490 & LaT & CPA & $c, 4553 t>a$ & V1518E & DIV S6 & LP & N/A \\
\hline $\begin{array}{l}\text { Mean } \\
( \pm S D)\end{array}$ & & $16 \pm 15$ & & $70 \pm 18$ & $390 \pm 80$ & $101 \pm 25$ & $521 \pm 86$ & & & & & & & \\
\hline
\end{tabular}

AVB, atrioventricular block; biT, bifid T-wave; CPA, cardiopulmonary arrest; D, domain; EoT, early onset T-wave; F, female; Fam, family member; GOF, gain-of-function; HR, heart rate; LaT, late-appearing T-wave; LP, likely pathogenic; LQT8, long QT syndrome type 8; M, male; N/A, no data; P, pathogenic; Pro, proband; QTc, corrected QT interval; QTp, QT peak interval; S, segment; TdP, Torsades de pointes; Tpe, T-wave peak to end; TWM, T-wave morphology; VF, ventricular fibrillation; VT, ventricular tachycardia.

tachycardia documented in 1 patient with syncope]; Table 1; Figure 4). One patient with EoT experienced syncope during a marathon. Notably, all documented fatal arrhythmic attacks (including TdP) were detected in the LaT group. In addition, 2 patients $(6.9 \%)$ with CPA in the LQT3 group exhibited LaT, and 6 of 13 patients (46\%) with LaT were symptomatic (Table 2). Only 1 female patient in the LQT3 group exhibited biT; this patient experienced TdP. There was no difference in the proportion of symptomatic patients between those exhibiting the LaT and other TWM patterns; however, only patients with LaT experienced CPA $(n=3$ in the LQT8 group, $n=2$ in the LQT3 group; 


\begin{tabular}{|c|c|c|c|c|c|c|c|c|c|c|c|c|c|c|}
\hline $\begin{array}{c}\text { Patient } \\
\text { no. }\end{array}$ & Status & $\begin{array}{c}\text { Age } \\
\text { (years) }\end{array}$ & Sex & $\begin{array}{c}\text { HR } \\
\text { (beats/ } \\
\text { min) }\end{array}$ & $\begin{array}{l}\text { QTp } \\
\text { (ms) }\end{array}$ & $\begin{array}{l}\text { Tpe } \\
\text { (ms) }\end{array}$ & $\begin{array}{l}\text { QTc } \\
\text { (ms) }\end{array}$ & TWM & Symptom & $\begin{array}{l}\text { Nucleotide } \\
\text { change }\end{array}$ & $\begin{array}{l}\text { Amino } \\
\text { acid } \\
\text { change }\end{array}$ & Location & $\begin{array}{l}\text { Pathoge- } \\
\text { nicity }\end{array}$ & $\begin{array}{c}\text { Func- } \\
\text { tional } \\
\text { proper- } \\
\text { ties }\end{array}$ \\
\hline 1 & Pro & 32 & $\mathrm{~F}$ & 68 & 400 & 120 & 550 & ЕoT & $\begin{array}{l}\text { Asymp- } \\
\text { tomatic }\end{array}$ & c. $337 \mathrm{~g}>\mathrm{a}$ & V113I & $\begin{array}{c}\text { N-termi- } \\
\text { nus }\end{array}$ & VUS & $N / A$ \\
\hline 2 & Pro & 12 & $M$ & 100 & 280 & 120 & 464 & Еот & $\begin{array}{l}\text { Asymp- } \\
\text { tomatic }\end{array}$ & c. $994 \mathrm{~g}>\mathrm{a}$ & А332T & $\begin{array}{l}\text { DI S5- } \\
\text { S6 pore }\end{array}$ & LP & $\mathrm{N} / \mathrm{A}$ \\
\hline 3 & Pro & 0 & $M$ & 129 & 360 & 100 & 638 & LaT & CPA & c. $1218 \mathrm{c}>\mathrm{a}$ & N406K & DI S6 & $P$ & GOF \\
\hline 4 & Pro & 5 & $\mathrm{~F}$ & 76 & 360 & 140 & 606 & EoT & Syncope & c. $1231 \mathrm{~g}>\mathrm{a}$ & V411M & DI S6 & $P$ & GOF \\
\hline 5 & Pro & 0 & M & 115 & 320 & 80 & 555 & LaT & $2: 1$ AVB & c. $1231 \mathrm{~g}>\mathrm{a}$ & V411M & DI S6 & $P$ & GOF \\
\hline 6 & Pro & 13 & $M$ & 68 & 350 & 80 & 477 & ЕoT & $\begin{array}{l}\text { Asymp- } \\
\text { tomatic }\end{array}$ & c.1324a>g & K442E & $\begin{array}{l}\text { DI-DII } \\
\text { linker }\end{array}$ & LP & $\mathrm{N} / \mathrm{A}$ \\
\hline 7 & Pro & 12 & M & 92 & 320 & 60 & 495 & LaT & Syncope & c. $2335 \mathrm{c}>\mathrm{a}$ & Q779K & $\begin{array}{l}\text { DII S2- } \\
\text { S3 linker }\end{array}$ & LP & N/A \\
\hline 8 & Pro & 12 & $M$ & 68 & 340 & 60 & 490 & Еот & $\begin{array}{l}\text { Asymp- } \\
\text { tomatic }\end{array}$ & c. $3250 \mathrm{~g}>\mathrm{a}$ & G1084S & $\begin{array}{l}\text { DII-DIII } \\
\text { linker }\end{array}$ & VUS & $\mathrm{N} / \mathrm{A}$ \\
\hline 9 & Fam & 4 & $\mathrm{~F}$ & 83 & 280 & 80 & 424 & ЕoT & $\begin{array}{l}\text { Asymp- } \\
\text { tomatic }\end{array}$ & c. $3250 \mathrm{~g}>\mathrm{a}$ & G1084S & $\begin{array}{l}\text { DII-DIII } \\
\text { linker }\end{array}$ & VUS & $\mathrm{N} / \mathrm{A}$ \\
\hline 10 & Pro & 0 & M & 55 & 620 & 120 & 719 & LaT & CPA & $\begin{array}{c}\text { c.4456- } \\
\text { 4458delttc }\end{array}$ & F1486del & $\begin{array}{l}\text { DIII-DIV } \\
\text { linker }\end{array}$ & $P$ & GOF \\
\hline 11 & Pro & 13 & $M$ & 60 & 400 & 120 & 520 & LaT & $\begin{array}{l}\text { Asymp- } \\
\text { tomatic }\end{array}$ & $\begin{array}{l}\text { c.4849- } \\
\text { 4851deltct }\end{array}$ & F1617del & $\begin{array}{c}\text { DIV } \\
\text { S3-4 }\end{array}$ & LP & N/A \\
\hline 12 & Fam & 38 & M & 55 & 360 & 120 & 474 & EoT & $\begin{array}{l}\text { Asymp- } \\
\text { tomatic }\end{array}$ & $\begin{array}{l}\text { c.4849- } \\
\text { 4851deltct }\end{array}$ & F1617del & $\begin{array}{l}\text { DIV } \\
\text { S3-4 }\end{array}$ & LP & N/A \\
\hline 13 & Pro & 6 & $\mathrm{~F}$ & 115 & 280 & 80 & 499 & biT & $\mathrm{TdP}$ & c. $4901 \mathrm{t}>\mathrm{c}$ & L1634P & DIV S4 & LP & N/A \\
\hline 14 & Pro & 18 & $F$ & 48 & 460 & 134 & 562 & EoT & Syncope & $\mathrm{c} .5236 \mathrm{~g}>\mathrm{a}$ & $\mathrm{A} 1746 \mathrm{~T}$ & DVIS6 & LP & N/A \\
\hline 15 & Fam & 10 & M & 84 & 300 & 110 & 488 & EoT & $\begin{array}{l}\text { Asymp- } \\
\text { tomatic }\end{array}$ & c. $5236 \mathrm{~g}>\mathrm{a}$ & $\mathrm{A} 1746 \mathrm{~T}$ & DVI S6 & LP & N/A \\
\hline 16 & Pro & 1 & $M$ & 95 & 480 & 120 & 680 & LaT & $\mathrm{TdP}$ & $c .5319 a>g$ & N1774D & $\begin{array}{c}\text { C-termi- } \\
\text { nus }\end{array}$ & $P$ & GOF \\
\hline 17 & Pro & 12 & $M$ & 68 & 360 & 120 & 500 & LaT & $\begin{array}{l}\text { Asymp- } \\
\text { tomatic }\end{array}$ & c. $5350 \mathrm{~g}>\mathrm{a}$ & E1784K & $\begin{array}{c}\text { C-termi- } \\
\text { nus }\end{array}$ & $P$ & GOF \\
\hline 18 & Pro & 14 & $M$ & 46 & 440 & 116 & 486 & LaT & $\begin{array}{l}\text { Asymp- } \\
\text { tomatic }\end{array}$ & c. $5350 \mathrm{~g}>\mathrm{a}$ & E1784K & $\begin{array}{c}\text { C-termi- } \\
\text { nus }\end{array}$ & $P$ & GOF \\
\hline 19 & Pro & 47 & $F$ & 51 & 460 & 82 & 518 & LaT & $\mathrm{TdP}$ & c. $5350 \mathrm{~g}>\mathrm{a}$ & E1784K & $\begin{array}{c}\text { C-termi- } \\
\text { nus }\end{array}$ & $P$ & GOF \\
\hline 20 & Fam & 6 & $M$ & 70 & 370 & 70 & 476 & LaT & $\begin{array}{l}\text { Asymp- } \\
\text { tomatic }\end{array}$ & c. $5350 \mathrm{~g}>\mathrm{a}$ & E1784K & $\begin{array}{c}\text { C-termi- } \\
\text { nus }\end{array}$ & $P$ & GOF \\
\hline 21 & Pro & 0 & $\mathrm{~F}$ & 125 & 260 & 60 & 520 & EoT & $\begin{array}{l}\text { Asymp- } \\
\text { tomatic }\end{array}$ & c. $5350 \mathrm{~g}>\mathrm{a}$ & E1784K & $\begin{array}{l}\text { C-termi- } \\
\text { nus }\end{array}$ & $P$ & GOF \\
\hline 22 & Fam & 23 & $\mathrm{~F}$ & 49 & 480 & 87 & 510 & LaT & $\begin{array}{l}\text { Asymp- } \\
\text { tomatic }\end{array}$ & c. $5350 \mathrm{~g}>\mathrm{a}$ & E1784K & $\begin{array}{c}\text { C-termi- } \\
\text { nus }\end{array}$ & $P$ & GOF \\
\hline 23 & Pro & 28 & M & 80 & 320 & 80 & 462 & EoT & Syncope & $\mathrm{c} .5350 \mathrm{~g}>\mathrm{a}$ & E1784K & $\begin{array}{l}\text { C-termi- } \\
\text { nus }\end{array}$ & $P$ & GOF \\
\hline 24 & Pro & 13 & $F$ & 60 & 540 & 80 & 620 & LaT & $\begin{array}{l}\text { Asymp- } \\
\text { tomatic }\end{array}$ & c. $5350 \mathrm{~g}>\mathrm{a}$ & E1784K & $\begin{array}{c}\text { C-termi- } \\
\text { nus }\end{array}$ & $P$ & GOF \\
\hline 25 & Pro & 7 & M & 90 & 340 & 84 & 518 & LaT & $\begin{array}{l}\text { Asymp- } \\
\text { tomatic }\end{array}$ & c.5384a>g & Y1795C & $\begin{array}{l}\text { C-termi- } \\
\text { nus }\end{array}$ & $P$ & GOF \\
\hline $\begin{array}{l}\text { Mean } \\
( \pm \text { SD })\end{array}$ & & $13 \pm 12$ & & $78 \pm 25$ & $379 \pm 89$ & $97 \pm 25$ & $530 \pm 72$ & & & & & & & \\
\hline
\end{tabular}

LQT3, long QT syndrome type 3; VUS, uncertain significance. Other abbreviations as in Table 1.

Figure 4).

Regarding the location of $C A C N A 1 C$ mutations, 5 of 9 patients $(55.6 \%)$ with LaT had $C A C N A 1 C$ mutations located in D-linker regions (Figure 2), and 4 of these 5 patients were symptomatic, including 2 cases of CPA ( $C A C N A 1 C$ variants $\mathrm{R} 412 \mathrm{M}$ and $\mathrm{S} 643 \mathrm{~F}$ ). Three patients with LaT had $C A C N A 1 C$ mutations located in the transmembrane region, and one mutation was located in the pore region. The remaining 1 mutation identified in a patient who experienced CPA was located in the D4-S6 transmembrane region ( $C A C N A 1 C-\mathrm{V} 1518 \mathrm{E})$. Moreover, 2 patients with $\mathrm{LaT}$ experienced TdP and both these patients had $C A C N A 1 C$-G402S (TS mutation) located in the D1S6 transmembrane region. Regarding other TWM groups, all mutations in patients exhibiting the biT pattern were in D-linker regions (Figure 2), whereas among patients with the EoT pattern, 1 mutation was located in the transmembrane region and the remaining 2 were in D-linker regions. In contrast, the locations of $S C N 5 A$ mutations were considerably scattered (Supplementary Figure). 


\begin{tabular}{|c|c|c|c|}
\hline & $\begin{array}{c}\text { LQT8 } \\
(n=25)\end{array}$ & $\begin{array}{c}\text { LQT3 } \\
(n=25)\end{array}$ & P-value \\
\hline No. males/females & $8 / 17$ & $16 / 9$ & 0.023 \\
\hline Mean age of diagnosis (years) & $16.0 \pm 14.8$ & $13.0 \pm 12.3$ & NS \\
\hline Mean HR (beats/min) & $70 \pm 18$ & $78 \pm 25$ & NS \\
\hline Mean QTc interval (ms) & $521 \pm 86$ & $530 \pm 72$ & NS \\
\hline \multicolumn{4}{|l|}{ T-wave morphology } \\
\hline \multicolumn{4}{|l|}{ EoT } \\
\hline No. patients & 5 & 11 & NS \\
\hline Age (years) & $17.2 \pm 13.1$ & $15.6 \pm 12.2$ & NS \\
\hline HR (beats/min) & $65 \pm 9$ & $78 \pm 21$ & NS \\
\hline QT peak (ms) & $354 \pm 24$ & $337 \pm 59$ & NS \\
\hline T-wave peak to end (ms) & $78 \pm 15$ & $100 \pm 29$ & NS \\
\hline QTc interval (ms) & $458 \pm 32$ & $502 \pm 53$ & NS \\
\hline \multicolumn{4}{|l|}{ LaT } \\
\hline No. patients & 17 & 13 & \\
\hline Age (years) & $14.4 \pm 15.3$ & $11.4 \pm 12.8$ & NS \\
\hline HR (beats/min) & $75 \pm 18$ & $75 \pm 27$ & NS \\
\hline QT peak (ms) & $377 \pm 70$ & $422 \pm 91$ & NS \\
\hline T-wave peak to end (ms) & $110 \pm 23$ & $95 \pm 22$ & NS \\
\hline QTc interval (ms) & $533 \pm 90$ & $557 \pm 80$ & NS \\
\hline \multicolumn{4}{|l|}{ bit } \\
\hline No. patients & 3 & 1 & \\
\hline Age (years) & $23.0 \pm 17.3$ & 6 & NA \\
\hline HR (beats/min) & $49 \pm 6$ & 115 & NA \\
\hline QT peak (ms) & $527 \pm 58$ & 280 & NA \\
\hline T-wave peak to end (ms) & $93 \pm 31$ & 80 & NA \\
\hline QTc interval (ms) & $556 \pm 93$ & 499 & NA \\
\hline Symptoms (n) & 9 & 10 & NS \\
\hline CPA & 3 & 2 & NS \\
\hline $\mathrm{TdP}$ & 2 & 3 & NS \\
\hline Syncope & 4 & 4 & NS \\
\hline 2:1 AV block & $1^{*}$ & 1 & NS \\
\hline
\end{tabular}

Unless indicated otherwise, data are presented as the mean \pm SD. ${ }^{*}$ This patient exhibited both $\mathrm{TdP}$ and 2:1 atrioventricular (AV) block. NA, not applicable. Other abbreviations as in Tables 1,2.

\section{TWM in LQT1 and LQT2 Patients}

Most LQTS patients are classified as either LQT1 or LQT2, so we checked TWM in 100 LQT1 and LQT2 patients each with pathogenic or likely pathogenic mutations. However, as reported previously, ${ }^{\mathbf{5}, \mathbf{6}}$ most of these patients exhibited characteristic T-wave patterns and no LaT was observed.

\section{Discussion}

This study investigated TWM in LQT8 and LQT3 patients, finding that the LaT pattern was seen in two-thirds of LQT8 patients, which correlated with several phenotypes. CACNAIC mutations in patients with the LaT pattern tended to be located in D-linker regions (Figure 2). However, in the LQT3 group, the LaT pattern was noted in one-third of patients, with the location of SCN5A mutations scattered and not correlated with TWM.

TWM patterns are useful to distinguish the genotype before genetic analysis:46,26 LQT1 exhibits early onset broad-based T-waves, LQT2 exhibits low-amplitude and bifid T-waves, and LQT3 exhibits long isoelectric ST segments with an LaT. Differences in LQTS-related ion channels and changes in action potential morphology could contribute to differences in TWM. ${ }^{5}$ In addition, in our LQTS database, most patients with LQT1-2 showed characteristic T-wave patterns ${ }^{5,6}$ and no LaT. In contrast, the LaT pattern was a major TWM in the LQT3 and LQT8 groups. Based on these results, TWM is helpful in estimating the LQTS genotype.

The LaT pattern has long been considered specific for LQT3 caused by SCN5A mutations. The cardiac $\mathrm{Na}^{+}$ current exhibits very fast kinetics (Figure 5A, Left), whereas LQTS-related $S C N 5 A$ mutants present long-lasting late $\mathrm{Na}^{+}$ current (Figure 5A, Right). ${ }^{27}$ The sustained inward currents may prolong the action potential duration (Figure 5D).

LQT8 results from gain-of-function mutations of $C A C N A 1 C$, encoding cardiac L-type calcium channels. A study on TS reported that the $C A C N A 1 C$-G406R mutation markedly slows the inactivation process. ${ }^{7,8}$ Functional analysis of several $C A C N A 1 C$ mutations in patients with LQT8 revealed mutations exerting 2 different types of gain-of-function, namely an increase in the peak current and a delayed inactivation (Figure 5B), ${ }^{9}$ both resulting in elevated late $\mathrm{Ca}^{2+}$ currents and thereby prolonging the action potential duration (Figure 5E). Because 2 genotype mutations partially share a similar mechanistic background, 
(A)

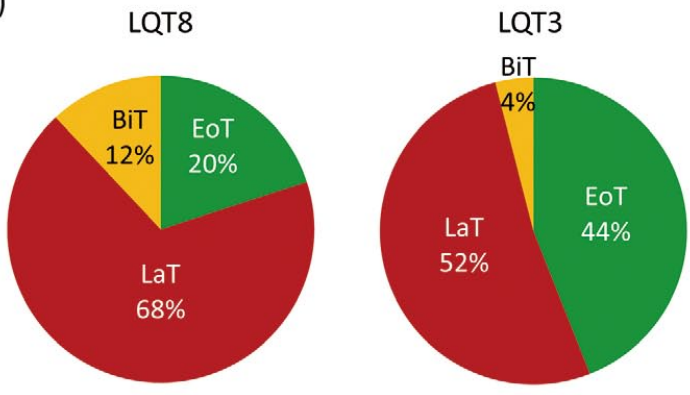

(C) QT peak intervals

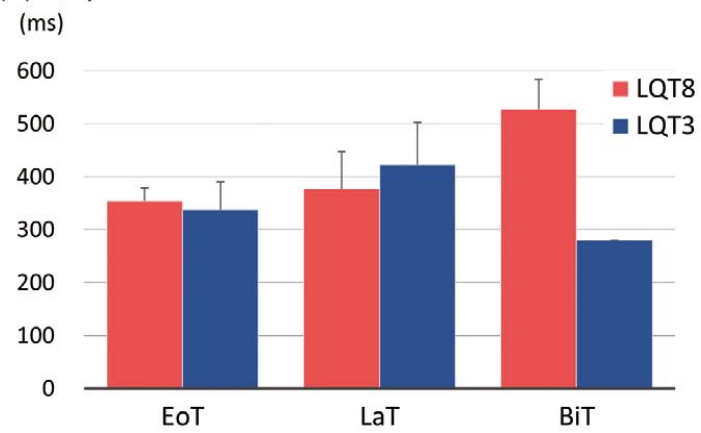

(B) QTc intervals

(ms)

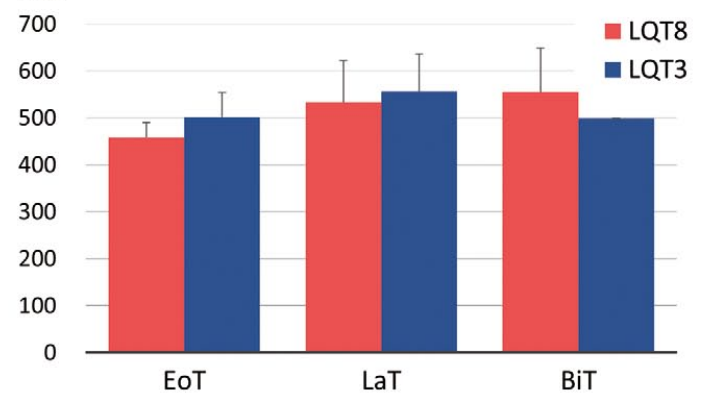

(D) Tpe intervals

(ms)

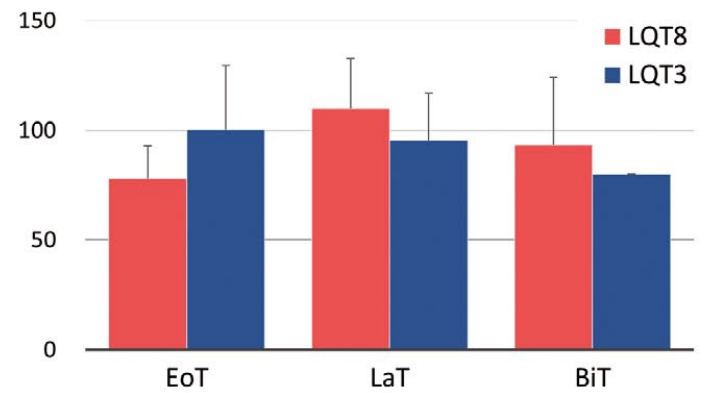

Figure 3. Comparisons between the long QT syndrome type 8 (LQT8) and type 3 (LQT3) groups. (A) The proportion of each T-wave morphology (TWM) in the LQT8 and LQT3 groups. EOT, early onset T-wave; LaT, late-appearing T-wave; biT, bifid T-wave. (B) Corrected QT (QTC), (C) QT peak, and (D) T-wave peak to end (Tpe) intervals in the LQT8 and LQT3 groups according to TWM. Data are the mean \pm SD.

\section{LQT8}

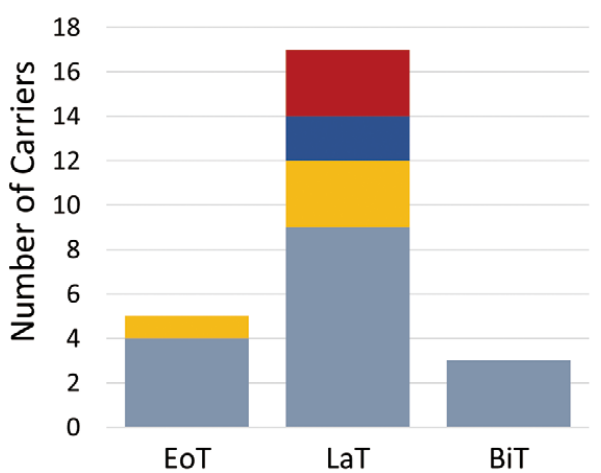

LQT3

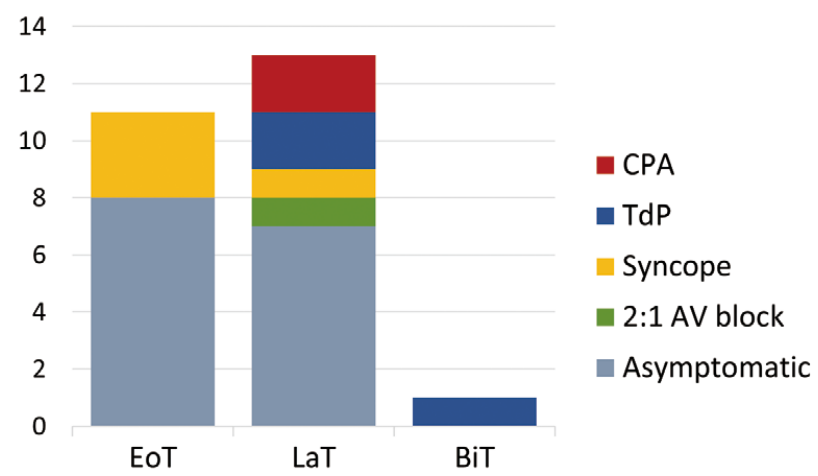

Figure 4. Comparison of the frequency of symptoms between the long QT syndrome type 8 (LQT8) and type 3 (LQT3) groups according to T-wave morphology. EoT, early onset T-wave; LaT, late-appearing T-wave; biT, bifid T-wave; AV, atrioventricular; CPA, cardiopulmonary arrest. TdP, Torsades de pointes.

these similarities in functional changes between LQT3 and LQT8 could result in similar TWMs.

In both the LQT3 and LQT8 groups in the present study, those with EoT exhibited a milder QTc prolongation than the other groups (Table 3; Figure 3B). In addition, ECG parameters, except for TWM, were not markedly different between the LQT3 and LQT8 groups. However, there was a higher proportion of female patients in the LQT8 group (Table 3). In terms of the sex difference, we failed to perform genetic screening of all family members, which could be attributed to entry bias.

Notably, in both the LQT8 and LQT3 groups, only carriers displaying the LaT pattern experienced CPA. Half the patients exhibiting the LaT pattern had symptoms or 
(A)

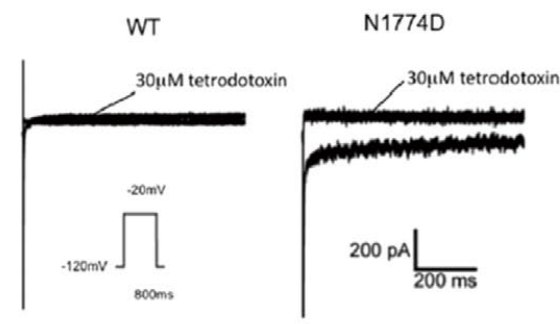

(B)

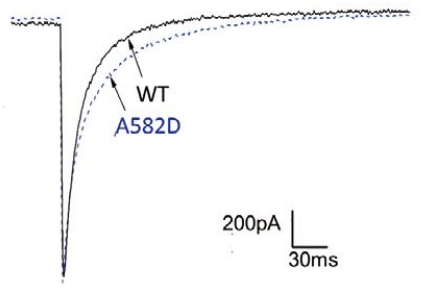

(C)

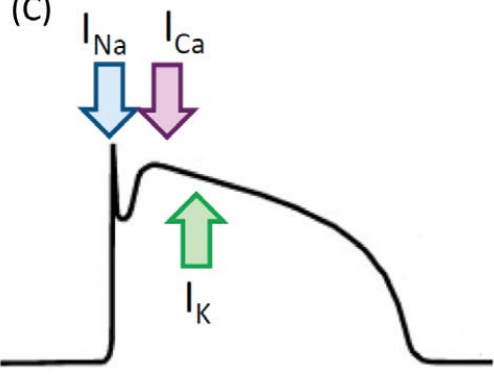

(D)

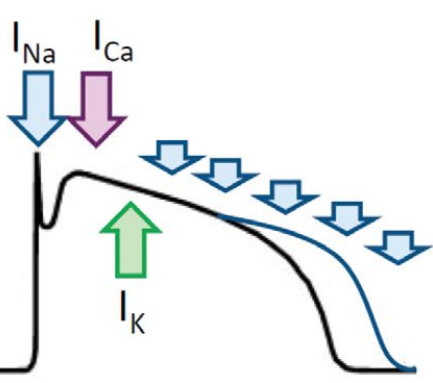

(E)

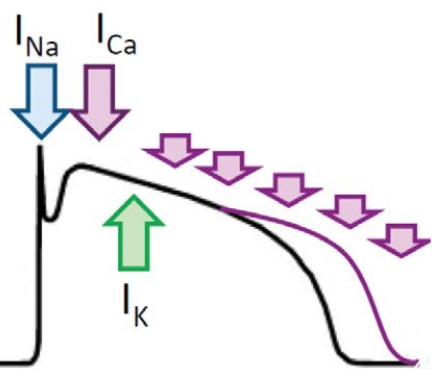

Figure 5. Functional analyses of mutations in the long QT syndrome type 8 (LQT8) and type 3 (LQT3) groups. (A) Functional analysis of mutant sodium channels (SCN5A-N1774D) shows the late current. ${ }^{27} \mathrm{WT}$, wild type. (B) The CACNA1C-A582D mutation inhibits its inactivation and exhibits an increase in the late current (blue arrow). ${ }^{9}$ (C) The normal action potential. (D) Continuous late current through mutant sodium channels (small blue arrows). (E) CACNA1C mutations causing LQT8 also results in prolongation of the action potential (pink arrows).

documented fatal arrhythmias, and one-third of these patients presented critical symptoms, such as CPA. In the other TWM groups, an LQT3 patient exhibiting biT had TdP. Furthermore, symptoms in EoT patients with LQT8 and LQT3 were mild, with most patients remaining asymptomatic. According to the 2015 ACMG standards and guidelines, ${ }^{24}$ all $C A C N A 1 C$ variants were categorized as pathogenic or likely pathogenic. Thus, we could evaluate the relationship between TWM and the severity of LQT8 phenotypes. Conversely, 3 patients with $S C N 5 A$ variants categorized as of uncertain significance showed EoT and were asymptomatic. However, all variants in patients with LaT in both the LQT8 and LQT3 groups were categorized as pathogenic.

Previous studies have reported LQT8 patients with $C A C N A 1 C$-P $857 \mathrm{R}^{10}$ and $\mathrm{I}_{1166 \mathrm{~T}^{12}}$ variants. The 3 patients with $C A C N A 1 C$-P857R showed EoT on ECG, and were asymptomatic. ${ }^{10}$ In contrast, the infant carrying $C A C N A I C$ I1166T clearly showed the LaT pattern and prominent QTc prolongation, as well as severe clinical phenotypes that were similar to TS (an implantable cardioverter-defibrillator was implanted in this patient, who had congenital heart disease and clinodactyly). ${ }^{12}$ Another study reported that patients with a $C A C N A 1 C$-L $762 \mathrm{~F}$ mutation had severe symptoms and also showed LaT. ${ }^{13}$

Clinical severity in patients with the LaT pattern could be related to late inward currents through voltage-gated $\mathrm{Na}^{+}$or $\mathrm{Ca}^{2+}$ channels. In the first report of classical TS (CACNA1C-G406R and G402S), mutant channels exhibited a severely slowed process of inactivation. ${ }^{8}$ However,
CACNA1C-R858H resulted in a different type of gain of function, merely increasing the peak calcium current. ${ }^{9}$ In addition, we found that $C A C N A I C-\mathrm{R} 412 \mathrm{M}$ exhibited a similar phenomenon in our following studies; significantly inhibited inactivation as classical TS (unpubl. data). Furthermore, the $C A C N A 1 C$-S643F mutant channel showed a gain of function similar to that of classical TS. ${ }^{14}$ Functional analysis of some mutations in the EoT group was performed, but no or only mild changes were noted in their calcium currents. With regard to cases of LQT3, the dysfunction in sodium channels was more complicated. For example, the $S C N 5 A$-E1784K mutation reportedly causes both LQTS and Brugada syndrome. ${ }^{28-30}$ The findings indicate that the presence of LaT could be a high risk for critical arrhythmic events in LQT8 and LQT3; the functional properties of each variant are described in Tables 1 and 2.

With regard to the topology of Cav1.2, 5 of 9 mutations resulting in LaT were located in D-linker regions. Four of these patients had severe symptoms, including 2 cases of $\mathrm{CPA}$. The remaining 2 symptomatic patients had syncope (CACNA1C-G406R and $\mathrm{R} 858 \mathrm{H})$ and non-sustained ventricular tachycardia (other CACNAIC-R858H). CACNA1C-G406R located in the DI-DII linker region is through to be the representative mutation of LQT8, also known as classical TS, which shows typical LaT.7,8

The position of $C A C N A 1 C$-G406 is reported to be associated with the voltage-dependent inactivation gate of Cav1.2. ${ }^{7}$ Severe disorders in the inactivation process may cause the LaT pattern. Interestingly, we identified 2 R518 
mutations in our cohort (R518C and R518H); although these mutations were located in the same position in the DI-II linker, their TWMs were different. Boczek et al undertook functional analysis of $C A C N A 1 C-\mathrm{R} 518 \mathrm{C} / \mathrm{H}$ mutations and reported that both resulted in significant gain of function due to delayed inactivation..$^{31}$ In addition, CACNA1C-R $518 \mathrm{C}$ was considered to affect trafficking. ${ }^{31}$ These differences may be related to the differences in TWM patterns between $C A C N A 1 C$ - $\mathrm{R} 518 \mathrm{C}$ and $\mathrm{R} 518 \mathrm{H}$.

In contrast, functional analysis of $C A C N A 1 C-\mathrm{R} 858 \mathrm{H}$ (DII-III linker) revealed that peak calcium current was elevated in mutant channels, thereby increasing the late lasting inward current. ${ }^{9}$ We hypothesized that DII-III linker mutations could result in the LaT pattern through a mechanism different from that of DI-II linker mutations. Nevertheless, severe changes in the calcium current may produce LaT and cause severe clinical symptoms. Conversely, the locations of mutations in Nav1.5 in patients in the LQT3 group were scattered, and there appears to be no particular tendency for any relationship between mutation locations and TWMs.

Almost all the LQT3 and LQT8 patients in this study had taken $\beta$-blockers (propranolol, nadolol, or bisoprolol). As additional treatment, a calcium channel blocker (verapamil) was used for LQT8 patients and a sodium channel blocker (mexiletine) was used for LQT3 patients. These medications could shorten the QT interval and prevent fatal arrhythmias, but the TWM remained unchanged.

With regard to study limitations, although TWMs are very similar in LQT3 and LQT8, this study could not show characteristics to distinguish them. To distinguish LQT8 from LQT3 based on ECG findings, more LQT8 patients need to be identified. In particular, the biT group appeared to have marked QTc prolongation and bradycardia; however, it was difficult to confirm these observations because these patients were rare. Although the number of patients in the LaT group was relatively large, the number was not adequate. We cannot say whether LaT is associated with a worse prognosis or not. The clinical characteristics of patients with LQT8 could not be adequately evaluated because of the small number of patients in this group. However, notably, LaT is a predictor of LQT3 or LQT8, which clinicians should consider while treating patients with LaT.

\section{Conclusions}

In this study, over half the LQT3 and LQT8 patients displayed LaT, and nearly half of those experienced symptoms or arrhythmic events. However, we could not differentiate LQT3 and LQT8 based on TWM patterns. Hence, further investigation is warranted to differentiate between LQT3 and LQT8 patients exhibiting LaT to optimize therapy.

\section{Acknowledgments}

The authors are grateful to the cardiologists who provided clinical information.

\section{Sources of Funding}

This study was supported, in part, by MEXT KAKENHI (Grant no. 15 H04818 [to M.H.]), 17K15999 [to M.F.], and 18K07875 [to S.O.]), from the Ministry of Education, Culture, Sports, Science, and Technology of Japan, as well as grants from the Ministry of Health, Labor and Welfare of Japan for Clinical Research on Intractable Disease (H27-032 [to M.H. and S.O.] and H29-055 [to S.O.]).

\section{Conflict of Interest}

None declared.

\section{References}

1. Horie M. Long QT syndrome presents not only as QT prolongation but also as abnormal T-wave morphology. Heart Rhythm 2017; 14: $1171-1172$.

2. Moss AJ, Schwartz PJ, Crampton RS, Tzivoni D, Locati EH, MacCluer J, et al. The long QT syndrome. Prospective longitudinal study of 328 families. Circulation 1991; 84: 1136-1144.

3. Priori SG, Wilde AA, Horie M, Cho Y, Behr ER, Berul C, et al. HRS/EHRA/APHRS Expert Consensus Statement on the Diagnosis and Management of Patients with Inherited Primary Arrhythmia Syndromes. Expert Consensus Statement on Inherited Primary Arrhythmia Syndromes: Document endorsed by HRS, EHRA, and APHRS in May 2013 and by ACCF, AHA, PACES, and AEPC in June 2013. Heart Rhythm 2013; 10: 1932-1963.

4. Schwartz PJ, Stramba-Badiale M, Crotti L, Pedrazzini M, Besana A, Bosi G, et al. Prevalence of the congenital long-QT syndrome. Circulation 2009; 120: 1761-1767.

5. Malfatto G, Beria G, Sala S, Bonazzi O, Schwartz PJ. Quantitative analysis of $\mathrm{T}$ wave abnormalities and their prognostic implications in the idiopathic long QT syndrome. J Am Coll Cardiol 1994; 23: 296-301.

6. Moss AJ, Zareba W, Benhorin J, Locati EH, Hall WJ, Robinson $\mathrm{JL}$, et al. ECG T-wave patterns in genetically distinct forms of the hereditary long QT syndrome. Circulation 1995; 92: 29292934.

7. Splawski I, Timothy KW, Sharpe LM, Decher N, Kumar P, Bloise $\mathrm{R}$, et al. $\mathrm{Ca}(\mathrm{V}) 1.2$ calcium channel dysfunction causes a multisystem disorder including arrhythmia and autism. Cell 2004; 119: $19-31$.

8. Splawski I, Timothy KW, Decher N, Kumar P, Sachse FB, Beggs AH, et al. Severe arrhythmia disorder caused by cardiac L-type calcium channel mutations. Proc Natl Acad Sci USA 2005; 102: $8089-8096$.

9. Fukuyama M, Wang Q, Kato K, Ohno S, Ding WG, Toyoda F, et al. Long QT syndrome type 8: Novel CACNA1C mutations causing QT prolongation and variant phenotypes. Europace 2014; 16: $1828-1837$.

10. Boczek NJ, Best JM, Tester DJ, Giudicessi JR, Middha S, Evans $\mathrm{JM}$, et al. Exome sequencing and systems biology converge to identify novel mutations in the L-type calcium channel, CACNA1C, linked to autosomal dominant long QT syndrome. Circ Cardiovasc Genet 2013; 6: 279-289.

11. Wemhöner K, Friedrich C, Stallmeyer B, Coffey AJ, Grace A, Zumhagen S, et al. Gain-of-function mutations in the calcium channel CACNA1C (Cav1.2) cause non-syndromic long-QT but not Timothy syndrome. J Mol Cell Cardiol 2015; 80: 186-195.

12. Boczek NJ, Miller EM, Ye D, Nesterenko VV, Tester DJ, Antzelevitch C, et al. Novel Timothy syndrome mutation leading to increase in CACNA1C window current. Heart Rhythm 2015; 12: $211-219$.

13. Landstrom AP, Boczek NJ, Ye D, Miyake CY, De la Uz CM, Allen HD, et al. Novel long QT syndrome-associated missense mutation, L762F, in CACNA1C-encoded L-type calcium channel imparts a slower inactivation tau and increased sustained and window current. Int J Cardiol 2016; 220: 290-298.

14. Ozawa J, Ohno S, Saito H, Saitoh A, Matsuura H, Horie M. A novel CACNA1C mutation identified in a patient with Timothy syndrome without syndactyly exerts both marked loss- and gainof-function effects. HeartRhythm Case Rep 2018; 4: 273-277.

15. Ye D, Tester DJ, Zhou W, Papagiannis J, Ackerman MJ. A pore-localizing CACNA1C-E1115K missense mutation, identified in a patient with idiopathic QT prolongation, bradycardia, and autism spectrum disorder, converts the L-type calcium channel into a hybrid nonselective monovalent cation channel. Heart Rhythm 2019; 16: 270-278.

16. Numa $\mathrm{S}$, Noda M. Molecular structure of sodium channels. Ann N Y Acad Sci 1986; 479: 338-355.

17. Catterall WA, Curtis BM. Molecular properties of voltage-sensitive calcium channels. Soc Gen Physiol Ser 1987; 41: 201-213.

18. Mikami A, Imoto K, Tanabe T, Niidome T, Mori Y, Takeshima H, et al. Primary structure and functional expression of the cardiac dihydropyridine-sensitive calcium channel. Nature 1989; 340: $230-233$.

19. Harada M, Suzuki H, Ohno S, Ozawa J, Saitoh A, Horie M. Dynamic QT changes in long QT syndrome type 8. Circ J 2019; 
83: 1614

20. Schwartz PJ. Idiopathic long QT syndrome: Progress and questions. Am Heart J 1985; 109: 399-411.

21. Schwartz PJ, Crotti L. QTc behavior during exercise and genetic testing for the long-QT syndrome. Circulation 2011; 124: 2181 2184 .

22. Millat G, Chanavat V, Créhalet H, Rousson R. Development of a high resolution melting method for the detection of genetic variations in long QT syndrome. Clin Chim Acta 2011; 412: 203-207.

23. Fukuyama M, Ohno S, Wang Q, Kimura H, Makiyama T, Itoh $\mathrm{H}$, et al. L-Type calcium channel mutations in Japanese patients with inherited arrhythmias. Circ J 2013; 77: 1799-1806.

24. Richards S, Aziz N, Bale S, Bick D, Das S, Gastier-Foster J, et al. Standards and guidelines for the interpretation of sequence variants: A joint consensus recommendation of the American College of Medical Genetics and Genomics and the Association for Molecular Pathology. Genet Med 2015; 17: 405-424.

25. Catterall WA. Structure and function of voltage-gated sodium and calcium channels. Curr Opin Neurobiol 1991; 1: 5-13.

26. Takenaka K, Ai T, Shimizu W, Kobori A, Ninomiya T, Otani H, et al. Exercise stress test amplifies genotype-phenotype correlation in the LQT1 and LQT2 forms of the long-QT syndrome. Circulation 2003; 107: 838-844.

27. Kato K, Makiyama T, Wu J, Ding WG, Kimura H, Naiki N, et al. Cardiac channelopathies associated with infantile fatal ventricular arrhythmias: From the cradle to the bench. J Cardiovasc Electrophysiol 2014; 25: 66-73.

28. Wei J, Wang DW, Alings M, Fish F, Wathen M, Roden DM, et al. Congenital long-QT syndrome caused by a novel mutation in a conserved acidic domain of the cardiac $\mathrm{Na}^{+}$channel. Circulation 1999; 99: 3165-3171.

29. Deschênes I, Baroudi G, Berthet M, Barde I, Chalvidan T, Denjoy I, et al. Electrophysiological characterization of SCN5A mutations causing long QT (E1784K) and Brugada (R1512 W and R1432G) syndromes. Cardiovasc Res 2000; 46: 55-65.

30. Makita N, Behr E, Shimizu W, Horie M, Sunami A, Crotti L, et al. The E1784K mutation in SCN5A is associated with mixed clinical phenotype of type 3 long QT syndrome. J Clin Invest 2008; 118: 2219-2229.

31. Boczek NJ, Ye D, Jin F, Tester DJ, Huseby A, Bos JM, et al. Identification and functional characterization of a novel CACNA1C-mediated cardiac disorder characterized by prolonged QT intervals with hypertrophic cardiomyopathy, congenital heart defects, and sudden cardiac death. Circ Arrhythm Electrophysiol 2015; 8: $1122-1132$.

\section{Supplementary Files}

Please find supplementary file(s); http://dx.doi.org/10.1253/circj.CJ-19-1101 